\title{
Conducta prosocial y autoatribuciones académicas en Educación Secundaria Obligatoria
}

\author{
Jesús Redondo ${ }^{1 *}$, Cándido J. Ingles ${ }^{2}$ y Jose M. García-Fernández ${ }^{3}$ \\ ${ }^{1}$ Facultad de Psicología. Universidad Pontificia Bolivariana de Bucaramanga, Colombia \\ ${ }^{2}$ Dpto. de Psicología de la Salud. Universidad Miguel Hernándę de Elche, España \\ ${ }^{3}$ Dpto. de Psicología Evolutiva y Didáctica. Universidad de Alicante, España
}

\begin{abstract}
Resumen: Este estudio presenta los resultados sobre la relación que existe entre las autoatribuciones académicas en lenguaje y matemáticas en una muestra de 2.022 estudiantes de Educación Secundaria de 12 a 16 años. Los adolescentes fueron seleccionados aleatoriamente de 20 escuelas urbanas y rurales en las provincias de Alicante y Murcia, España. La conducta prosocial fue codificada con el Teenage Inventory of Social Skills y las autoatribuciones académicas fueron medidas mediante la Escala de Atribución Causal de Sydney (Sydney Attribution Scale, SAS; Marsh, 1984). El 17.35\% de estudiantes de ESO fueron identificados como prosociales. Los chicos de $2^{\circ}$ de ESO y las chicas de $4^{\circ}$ de ESO presentaron la menor y mayor prevalencia puntual de conducta prosocial, respectivamente. Respecto a la asignatura de lenguaje, los estudiantes prosociales atribuyen significativamente el éxito a la capacidad, el esfuerzo y, en menor medida, a causas externas. En cuanto a la asignatura de matemáticas, los estudiantes prosociales atribuyeron el éxito significativamente más al esfuerzo y significativamente menos a causas externas, mientras que atribuyeron el fracaso significativamente más a la falta de esfuerzo. Además, los datos han permitido crear un modelo de regresión logística que permite hacer estimaciones correctas respecto a la probabilidad de éxito académico en matemáticas, en lenguaje y en todas las asignaturas aprobadas en estudiantes prosociales de E.S.O. a partir de las puntuaciones en atribuciones académicas.

Palabras clave: Conducta prosocial; autoatribuciones académicas; Educación Secundaria Obligatoria.
\end{abstract}

\section{Introducción}

En la adolescencia se producen un gran número de cambios biológicos, psicológicos y sociales. Mientras que algunos adolescentes atraviesan con éxito esta etapa, otros se encuentran con importantes dificultades (Carlo, Fabes, Laible y Kupanoff, 1999). El grupo de iguales va ganando una mayor importancia a medida que el niño avanza hacia la adolescencia, constituyéndose en un elemento esencial para su adecuado desarrollo emocional y social.

Existen estudios correlacionales que indican que las distintas manifestaciones de la conducta prosocial tales como las habilidades sociales, la tendencia empática, ser cooperador, complaciente y dispuesto a compartir, la conducta social apropiada en clase y el cumplimiento de normas en el aula y en la escuela en general (responsabilidad social) están relacionadas positivamente con el logro académico (Feshbach y Feshbach, 1987; Green, Forehand, Beck y Vosk, 1980; Entwisle, Alexander, Pallas y Cadigan, 1987; Wentzel, 1989, 1991b, 1993; Inglés et al., 2009; García-Fernández et al., 2010; Carrasco y Trianes, 2010).

Diversos investigadores han resaltado la semejanza de estilos atribucionales en el área social y académica (por

\footnotetext{
* Dirección para correspondencia [Correspondence address]:

Jesús Redondo Pacheco. Facultad de Psicología. Escuela de Ciencias Sociales. Universidad Pontificia Bolivariana. Kilometro 7, vía Piedecuesta. Bucaramanga (Santander). Colombia.

E-mail: jesus.redondo@upb.edu.co
}

Title: Prosocial behavior and academic self-attributions in Secondary Education.

Abstract: This study presents the findings of the relationship between prosocial behavior and academic self-attributions in language and math in a sample of 2022 students in secondary education among 12 to 16 year olds. Teenagers were randomly selected from 20 urban and rural schools in the provinces of Alicante and Murcia, Spain. Prosocial behavior was coded with the Teenage Inventory of Social Skills and academic self-attributions was measured by the Sydney Attribution Scale, SAS (Marsh, 1984). 17.35\% of the students from ESO were identified as prosocial. Boys from second grade and girls from fourth grade had the lowest and highest prevalence of prosocial behavior, respectively. Regarding the subject of language, students prosocial significantly attributed the success to ability, effort, and to a lesser extent, to external causes. As for the mathematics, the students attributed the success prosocial significantly more effort and significantly less to external causes, whereas significantly more failure attributed to lack of effort. Furthermore, data have created a logistic regression model that allows for accurate estimates about the likelihood of academic success in math, language and all subjects passed at ESO prosocial from scores on academic responsibilities.

Key words: Prosocial behaviour; academic self-attributions; secondary education.

ejemplo, Diener y Dweck, 1978; Dweck, 1975; Ford, 1996; Goetz y Dweck, 1980; Weiner, 1986, 2000). Estos autores indicaron que, tanto en situaciones académicas como sociales, los estudiantes que atribuyen sus fracasos académicos y rechazos sociales a la falta de capacidades tienden a abandonar el esfuerzo y la persistencia hacia el éxito en lugar de centrarse en minimizar el fracaso. Por el contrario, aquellos que se centran sobre su esfuerzo u otros factores controlables tienden a mostrar mayor persistencia en situaciones sociales y académicas.

Por otra parte, también se ha comprobado que las atribuciones de intencionalidad juegan un papel importante en la motivación social. Por ejemplo, cuando los niños agresivos atribuyen sistemáticamente hostilidad a sus iguales, incluso en situaciones accidentales, éstos parecen abandonar metas prosociales y en su lugar adoptan las metas de represalias y venganzas (Dodge, 1980; Dodge y Fram, 1982; Erdley y Asher, 1993). Así, las atribuciones de los estudiantes pueden influir de forma significativa sobre la motivación social y académica de los adolescentes y, a la inversa, las metas alcanzadas por los jóvenes pueden afectar a las atribuciones causales que realizan. Cuando los niños y adolescentes se preocupan excesivamente por probarse así mismos intelectual o socialmente, es más probable que perciban el fracaso o el rechazo como una crítica hacia sí mismos (por ejemplo, su escasa inteligencia o su personalidad inestable; Elliot y Dweck, 1988). Por el contrario, cuando los niños se preocupan en exceso por dominio, el control y la autodefensa es 
más probable que interpreten las acciones de otros como provocaciones (Erdley, 1996).

Los estudiantes tienen ciertas teorías implícitas o autoconcepciones que afectan a la elección de un tipo u otro de orientación motivacional. Por ejemplo, algunos estudiantes creen que su inteligencia es una entidad fija e inmutable (teoría de la entidad), mientras que otros creen que es una cualidad modificable que pueden incrementar mediante su esfuerzo (teoría incremental). Cuando los estudiantes piensan que su inteligencia o capacidad es fija, éstos se orientan extrínsecamente puesto que su objetivo principal es obtener juicios positivos de los demás. Por el contrario, cuando los estudiantes mantienen una teoría incremental, tienden a orientarse más intrínsecamente puesto que su objetivo es aumentar sus capacidades y habilidades. Además, Erdely, Cain, Loomis, Dumas-Hines y Dweck (1997) encontraron que estas relaciones también se mantienen en el contexto social. Como en el contexto académico, la orientación hacia la obtención de juicios positivos, y evitación de evaluaciones negativas, por parte de iguales, profesores y padres conduce a una mayor vulnerabilidad hacia autoatribuciones negativas y a una disminución de la persistencia en situaciones sociales complicadas tales como el rechazo social.

Las investigaciones realizadas por Dweck y sus colaboradores sobre atribuciones (Diener y Dweck, 1978; Goetz y Dweck, 1980), teorías implícitas y orientaciones motivacionales (Dweck y Leggett, 1988; Elliot y Dweck, 1988) han revelado que el patrón cognitivo-motivacional presente en el contexto escolar es análogo al que los niños y adolescentes presentan en el contexto social (Dweck, 1996). Autoatribuciones similares conducen a una respuesta no persistente de indefensión en ambos dominios. Asimismo, metas y teorías implícitas similares parecen configurar estas atribuciones y respuestas en ambos dominios. Esta es la formulación, por tanto, que los investigadores deberían adoptar entre ambos dominios (y entre literaturas) para establecer patrones generales de motivación y adaptación (Dweck, 1996; Weiner, 2000).

Finalmente, las aportaciones de diversos investigadores (Erdley, 1996; Junoven, 1996; Schunck y Zimmerman, 1996) indican que para alcanzar las metas deseadas, los estudiantes deben presentar cierto grado de conocimiento estratégico, es decir, deben conocer qué estrategias son las más adecuadas para conseguir lo que pretenden. Además, estas estrategias son muy cambiantes con la edad. Así, los niños pequeños que valoran la aprobación y aceptación de sus iguales, deben saber que éstos valoran su esfuerzo académico en gran medida. Sin embargo, los adolescentes que también pretenden ser aceptados por sus compañeros deben saber que éstos no aprueban el esfuerzo académico en la misma medida que cuando se es más pequeño. Por tanto, si los adolescentes desean mantener la aceptación de sus compañeros deben aprender a ocultar su esfuerzo y a proteger el mismo no desafiando intelectualmente a sus iguales (Dweck, 1996).

El modelo dinámico de metas establecido por Dweck (1996) indica que los estudiantes se introducen en las situa- ciones con determinadas creencias de eficacia, teorías implícitas y valores que hacen que ciertas metas u orientaciones motivacionales prevalezcan sobre otras. Estas metas iniciales pueden afectar a la forma en la que los estudiantes interpretan una determinada situación, debido al tipo de atribuciones causales que establecen. Este modelo puede ilustrarse adecuadamente mediante la descripción de estudiantes agresivos y tímidos o retraídos, quienes, junto a los estudiantes prosociales, conforman los tres estilos de respuesta interpersonal más frecuentes en la adolescencia (García-Fernández et al., 2005).

Los estudiantes agresivos, al igual que los prosociales y los tímidos o retraídos se introducen en o incorporan a las situaciones con el fin de alcanzar distintas metas, aunque las metas de dominancia y control parecen predominar en éstos (Crick y Ladd, 1990; Erdley y Asher, 1993). Este interés en mantener el dominio y control puede hacerles especialmente vulnerables a las peleas y provocaciones y puede conducirles a percibir, sesgadamente, una intención hostil por parte de los otros, lo que, a su vez, puede incrementar su ira y conductas agresivas (Dodge, 2006). En consonancia con estos resultados, Hull, Van Treuren y Propsom (1998) encontraron que los estudiantes universitarios caracterizados por puntuaciones elevadas en dureza y agresividad se caracterizaron por su tendencia a realizar atribuciones internas, estables y globales (capacidad) en situaciones de éxito, mientras que tendieron a realizar atribuciones externas, inestables y específicas (suerte, dificultad de la tarea, manía del profesor) en situaciones de fracaso. Asimismo, los resultados de los estudios previos coinciden, directa o indirectamente, con los hallados de numerosas investigaciones recientes. Concretamente, diversos investigadores han hallado que bajas puntuaciones en autoconcepto académico se relacionan con una mayor probabilidad de emisión de conductas violentas o agresivas en la escuela (Clark, Dogan y Akbar, 2003; Taylor, Davis y Malachuk, 2007; Vermeiren, Bogaerts, Ruchkin, Deboutte y Schwab-Stone, 2004). Además, el fracaso escolar es un predictor significativo de la conducta agresiva (Martens y Witt, 2004), y a la inversa; la conducta antisocial es un predictor muy potente del fracaso escolar (Chen, Chen y Kaspar, 2001; Chen, Rubin, Li y Li, 2001; Raine et al., 2006; Vandiver, 2001).

La literatura psicológica ha estudiado ampliamente las influencias motivacionales y contextuales de la conducta prosocial (por ejemplo, Batson, 1990; Wentzel, 1994). Sin embargo, esta literatura no está bien organizada ya que los procesos motivacionales son generalmente estudiados de forma aislada más que como componentes de patrones motivacionales más amplios. Además, conceptos clave tales como el de empatía son definidos de manera distinta por los investigadores.

La evidencia empírica ha demostrado que los valores y comportamientos asociados a la conducta socialmente responsable facilitan el aprendizaje escolar y el desarrollo intelectual de los estudiantes (Wentzel, 1989, 1991, 1993; Gutiérrez, Escartí y Pascual, 2011), mostrando que la conexión 
entre los dominios académico y social es de naturaleza motivacional. Concretamente, las metas, emociones y creencias de control personal que apoyan la conducta socialmente responsable facilitan el apego hacia la escuela y el esfuerzo en las tareas escolares (Ford, 1996; Inglés et al., 2011).

\section{Método}

\section{Participantes}

Se realizó un muestreo aleatorio por conglomerados (zonas geográficas de las provincias de Murcia y Alicante: centro, norte, sur, este y oeste). Con el fin de que todas las zonas geográficas estuvieran representadas se seleccionaron aleatoriamente 20 centros de áreas rurales y urbanas, 14 públicos y 6 privados. Cada zona geográfica estuvo representada por una media de dos centros. Una vez determinados los centros del estudio, se seleccionaron aleatoriamente cuatro aulas computándose aproximadamente 120 sujetos por centro.

El total de sujetos reclutados fue de 2.267 estudiantes de $1^{\circ}$ a $4^{\circ}$ de E.S.O. (error muestral $=.02$ ), de los que 116 $(5.12 \%)$ fueron excluidos por errores $\mathrm{u}$ omisiones en sus respuestas, por no alcanzar un nivel satisfactorio en la escala de Sinceridad del Cuestionario de Personalidad de Eysenck (EPQ), por no obtener el consentimiento de los padres para participar en la investigación o por ser extranjeros con un déficit elevado en el dominio de la lengua española. La muestra definitiva se compuso de 2.022 estudiantes, con un rango de edad de 12 a 16 años $(M=13.81 ; D T=1.35)$. El $13.7 \%$ de los estudiantes fueron repetidores. La composición étnica de la muestra fue la siguiente: $88.9 \%$ españoles, $6.34 \%$ latinoamericanos, $3.37 \%$ resto Europa, .5\% árabes y $.64 \%$ asiáticos. La Tabla 1 muestra la distribución de los sujetos por género y curso académico.

Tabla 1. Número (y porcentaje) de sujetos de la muestra clasificados por género y curso.

\begin{tabular}{lccccc}
\multicolumn{6}{c}{ género y curso. } \\
\hline Varones & $1^{\circ}$ E.S.O. & $2^{\circ}$ E.S.O. & $3^{\circ}$ E.S.O. & $4^{\circ}$ E.S.O. & Total \\
& $(15.3 \%)$ & 251 & 260 & 213 & 1033 \\
Mujeres & 267 & 254 & 242 & 226 & 989 \\
& $\left(13.2^{\circ} \%\right)$ & $(12.6 \%)$ & $\left(12.6^{\circ} \%\right)$ & $(11.2 \%)$ & $(48.9 \%)$ \\
Total & 576 & 505 & 502 & 439 & 2022 \\
& $(28.5 \%)$ & $(25 \%)$ & $(24.8 \%)$ & $(21.7 \%)$ & $(100 \%)$ \\
\hline
\end{tabular}

Por medio de la prueba Chi-cuadrado de homogeneidad de la distribución de frecuencias, se comprobó que no existían diferencias estadísticamente significativas entre los ocho grupos de género x curso $\left(\chi^{2}=3.15 ; p=.368\right)$.

\section{Instrumentos}

Inventario de Habilidades Sociales para Adolescentes (TISS, Teenage Inventory of Social Skills; Inderbitzen y Foster, 1992).El TISS evalúa la conducta prosocial y agresiva de los ado- lescentes en las relaciones con sus iguales. Consta de 40 ítems agrupados en dos escalas: Conducta Prosocial y Conducta Antisocial. Los ítems se valoran mediante una escala Likert de seis puntos $(1=$ no me describe nada; $6=$ me describe totalmente $)$. Ofrece dos puntuaciones, una de conducta prosocial y otra de conducta antisocial, las cuales se obtienen sumando los valores asignados por los sujetos a los ítems que componen ambas dimensiones. Puntuaciones altas indican elevada conducta prosocial y agresiva.

Las propiedades psicométricas del TISS, informadas por Inderbitzen y Foster (1992) en población adolescente norteamericana, fueron satisfactorias y semejantes a las halladas en muestras de adolescentes españoles (Inglés, Hidalgo et al., 2003), siendo uno de los cuestionarios de habilidades sociales con mejores garantías psicométricas en población adolescente (Inglés, Méndez, Hidalgo, Rosa y Estévez, 2003).

Escala de Atribución Causal de Sydney (Sydney Attribution Scale, SAS; Marsh, 1984).- El enfoque disposicional de las diferencias individuales, a diferencia del enfoque situacional, asume cierta tendencia estable a atribuir éxito o fracaso a determinadas causas por parte de un mismo estudiante en distintas situaciones escolares, es decir, un estilo atribucional generalmente constante en el contexto académico (Marsh, 1984; Marsh, Cairns, Relich, Barnes y Debus, 1984). A partir de estos postulados teóricos, Marsh y sus colaboradores elaboraron la Escala de Atribución de Sydney (SAS).

El objetivo de esta escala es evaluar las percepciones de los sujetos sobre las causas de su éxito o fracaso académico. La versión original de esta prueba consta de 24 situaciones que incluyen: dos áreas académicas (matemática y verbal), tres tipos de causas (habilidad, esfuerzo, causas externas) y dos resultados con valencia contraria (situaciones que implican éxito y situaciones que implican fracaso). De esta forma, la escala queda constituida por 72 ítems a las que los sujetos deben responder según una escala de cinco puntos $(1=$ Falso $5=$ Verdadero) .

Las investigaciones desarrolladas por Marsh (1984) y Marsh et al. (1984) revelaron que las diferencias individuales en autoatribuciones no pueden explicarse únicamente a través de las tres dimensiones bipolares propuestas por Weiner (1974). Así, encontraron un apoyo claro en cuanto a la diferenciación de atribuciones según el resultado académico (éxito o fracaso), la causa implicada (habilidad, esfuerzo, causas externas) y el contenido académico (verbal o numérico), concluyendo que las tendencias disposicionales en los procesos de atribución son específicas del resultado, la causa percibida y el contenido académico. Además, desde esta perspectiva se entiende que las dimensiones de éxito y fracaso están íntimamente relacionadas.

Las propiedades psicométricas de esta escala hacen de la SAS una medida excelente para evaluar autoatribuciones académicas en estudiantes de Educación Primaria y Secundaria. Diferentes investigaciones arrojan índices de consistencia interna para las escalas que componen el SAS plenamente satisfactorios, mostrando, además, una estructura fac- 
torial multidimensional e interrelacionada, coherente con los postulados teóricos de partida. En este sentido, cabe destacar que, aunque las investigaciones realizadas han abordado el estudio de la estructura interna de la SAS mediante análisis factorial de ejes principales iterados con rotación oblicua, éste ha sido aplicado, según el estudio, sobre las puntuaciones del total de ítems que componen la prueba, sobre las puntuaciones de los ítems relativos al éxito y al fracaso académico por separado, o sobre las puntuaciones totales en las seis o doce dimensiones hipotetizadas teóricamente por los autores originales. La Tabla 2 ofrece, esquemáticamente, las soluciones factoriales y los coeficientes de consistencia interna (alfa de Cronbach) hallados a partir de muestras de estudiantes de Educación Primaria y Secundaria de Australia (Craven, Marsh y Debus, 1991; Marsh, 1984), Chile (PérezVillalobos, González-Pienda, Núñez y Díaz-Mújica, 1996), España (González-Pienda et al., 2002; González-Pumariega, González-Pienda y Núñez, 1996; Núñez y González-Pienda, 1994), Estados Unidos (Bell et al., 1994; Bempechat, Graham y Jiménez, 1999; Bempechat, Nakkula, Wu y Ginsburg, 1996; Cooley, Beaird y Ayres, 1994) y Filipinas (Watkins y Gutiérrez, 1990).

En este estudio se emplearon seis puntuaciones para lenguaje y seis puntuaciones para matemáticas, correspondientes a las seis escalas generales: seis puntuaciones para lenguaje y seis puntuaciones para matemáticas, correspondientes a las seis escalas generales: Atribución del Éxito a la Capacidad/Habilidad, Atribución del Éxito al Esfuerzo, Atribución del Éxito a Causas Externas, Atribución del Fracaso a la Capacidad/Habilidad, Atribución del Fracaso al Esfuerzo y Atribución del
Fracaso a Causas Externas. De esta forma cada escala quedó constituida por un total de 12 ítems. Los coeficientes de consistencia interna ( $\alpha$ de Cronbach) para la asignatura de leguaje fueron: .79 (Éxito/Esfuerzo), .70 (Éxito/Habilidad), .52 (Exito/Causas Externas), .76 (Fracaso/Habilidad), .72 (Fracaso/Esfuerzo) y .51 (Fracaso/Causas Externas). Los índices de homogeneidad para la asignatura de matemáticas fueron: .81 (Éxito/Esfuerzo), .73 (Éxito/Habilidad), .59 (Éxito/Causas Externas), .80 (Fracaso/Habilidad), .77 (Fracaso/Esfuerzo) y .53 (Fracaso/Causas Externas).

\section{Resultados}

La Tabla 2 presenta las puntuaciones medias (y desviaciones típicas) de los estudiantes prosociales y no prosociales de E.S.O. en las escalas de la SAS. Respecto a la asignatura de lenguaje, los estudiantes prosociales atribuyen significativamente más que su iguales no prosociales el éxito a la capacidad, el esfuerzo y, en menor medida, a causas externas. Por el contrario, los estudiantes no prosociales atribuyen el fracaso en esta materia a su escasa capacidad en mayor medida que sus iguales no prosociales. En cuanto a la asignatura de matemáticas, los estudiantes prosociales, comparados con sus iguales no prosociales, atribuyeron el éxito significativamente más al esfuerzo y significativamente menos a causas externas, mientras que atribuyeron el fracaso significativamente más a la falta de esfuerzo. Sin embargo, la magnitud de las diferencias halladas fue pequeña en todos los casos.

Tabla 2. Diferencias entre sujetos prosociales y no prosociales en las escalas de la SAS.

\begin{tabular}{|c|c|c|c|c|c|}
\hline \multirow[t]{2}{*}{ Escalas SAS } & \multirow{2}{*}{$\begin{array}{c}\text { Prosociales } \\
M(D T)\end{array}$} & \multirow{2}{*}{$\begin{array}{c}\text { No prosociales } \\
M(D T)\end{array}$} & \multicolumn{3}{|c|}{$\begin{array}{l}\text { Significación estadística y } \\
\text { magnitud de las diferencias }\end{array}$} \\
\hline & & & $t_{(2020)}$ & $p$ & $d$ \\
\hline Éxito en lenguaje atribuido a la capacidad & $4.10(0.85)$ & $3.69(0.91)$ & 8.07 & .000 & .46 \\
\hline Éxito en lenguaje atribuido al esfuerzo & $3.74(0.87)$ & $3.50(0.82)$ & 7.04 & .000 & .40 \\
\hline Éxito en lenguaje atribuido a causas externas & $3.41(0.76)$ & $3.27(0.75)$ & 3.17 & .002 & .18 \\
\hline Fracaso en lenguaje atribuido a la capacidad & $1.94(0.89)$ & $2.18(0.88)$ & 4.70 & .000 & -.27 \\
\hline Fracaso en lenguaje atribuido al esfuerzo & $2.68(.71)$ & $2.74(.72)$ & -1.32 & n.s. & \\
\hline Fracaso en lenguaje atribuido a causas externas & $3.35(.67)$ & $3.32(.68)$ & .67 & n.s. & \\
\hline Éxito en matemáticas atribuido a la capacidad & $2.95(1.15)$ & $3.02(1.10)$ & -1.15 & n.s. & \\
\hline Éxito en matemáticas atribuido al esfuerzo & $3.71(.85)$ & $3.37(.87)$ & 6.66 & .000 & .39 \\
\hline Éxito en matemáticas atribuido a causas externas & $2.78(.77)$ & $2.94(.73)$ & 3.84 & .000 & -.21 \\
\hline Fracaso en matemáticas atribuido a la capacidad & $2.69(1.09)$ & $2.59(1.01)$ & 1.58 & n.s. & \\
\hline Fracaso en matemáticas atribuido al esfuerzo & $2.87(.69)$ & $2.78(.71)$ & 2.12 & .034 & .12 \\
\hline Fracaso en matemáticas atribuido a causas externas & $3.15(.67)$ & $3.13(.65)$ & .54 & n.s. & \\
\hline
\end{tabular}

Nota. n.s. $=$ no significativo

Los resultados de este estudio indican que los estudiantes prosociales atribuyen significativamente más que los estudiantes no prosociales el éxito en lenguaje a la capacidad y al esfuerzo y, en menor medida, a causas externas, el éxito en matemáticas al esfuerzo y el fracaso en matemáticas a la falta de esfuerzo. Por el contrario, los estudiantes prosociales atribuyen significativamente menos que los estudiantes no prosociales el éxito en matemáticas a causas externas y el fracaso en lenguaje a la baja capacidad.
Las variables dependientes correspondientes al rendimiento en las asignaturas de matemáticas y lenguaje en la muestra de estudiantes prosociales fueron dicotomizadas, categorizándolas en éxito $(n=202)$ y fracaso $(n=74)$ en matemáticas y éxito $(n=231)$ y fracaso $(n=46)$ en lenguaje. Los datos permitieron crear dos modelos de regresión logística que permiten hacer estimaciones correctas respecto a la probabilidad de éxito en matemáticas y en lenguaje en estudiantes prosociales de E.S.O. a partir de las puntuaciones en 
atribuciones académicas. La Tabla 3 muestra los pasos seguidos por los modelos en la introducción de las variables explicativas que han resultado significativas para la probabilidad de éxito matemático y lingüístico. En este sentido, el estadístico $\mathrm{R}^{2}$ de Nagelkerke estima un valor de ajuste de .27 para el modelo de éxito en matemáticas y de .11 para el de lenguaje.

Tabla 3. Pruebas omnibus y ajuste sobre los coeficientes del modelo de matemáticas y lenguaje.

\begin{tabular}{|c|c|c|c|c|c|}
\hline & Variables & $\chi^{2}$ & $g l$ & $p$ & $\mathrm{R}^{2}$ de Nagelkerke \\
\hline \multicolumn{6}{|c|}{ Matemáticas } \\
\hline \multirow[t]{2}{*}{ Paso 1} & Fracaso matemáticas atribuido a la capacidad & 57.78 & 1 & .000 & \\
\hline & Modelo & 57.78 & 1 & .000 & .27 \\
\hline \multicolumn{6}{|l|}{ Lenguaje } \\
\hline \multirow[t]{2}{*}{ Paso 1} & Éxito lenguaje atribuido al esfuerzo & 11.77 & 1 & .001 & \\
\hline & Modelo & 11.77 & 1 & .001 & .07 \\
\hline \multirow[t]{2}{*}{ Paso 2} & Fracaso matemáticas capacidad & 6.50 & 1 & .011 & \\
\hline & Modelo & 18.28 & 2 & .000 & .11 \\
\hline
\end{tabular}

Nota. $g l=$ grados de libertad. $p=$ probabilidad.

El modelo creado para la variable dependiente éxito matemático permite una estimación correcta del 79\% de los casos, $\chi^{2}=57.78 ; p<.001$, entrando a formar parte de la ecuación la variable predictora atribución del fracaso en matemáticas a la baja capacidad. El modelo predictivo del éxito lingüístico permite una estimación correcta del $82.7 \%$ de los casos, $\chi^{2}=18.28 ; p<.001$, entrando a formar parte de la ecuación las variables independientes la atribución del éxito en lenguaje al esfuerzo y atribución del fracaso en matemáticas a la baja capacidad (ver Tabla 4).

Tabla 4. Número y porcentaje de casos clasificados correctamente en la predicción del éxito en matemáticas.

\begin{tabular}{|c|c|c|c|}
\hline \multirow[t]{2}{*}{ Observado } & \multicolumn{2}{|c|}{ Pronosticado } & \multirow{2}{*}{$\begin{array}{l}\% \text { Casos } \\
\text { acertados }\end{array}$} \\
\hline & Suspenso & Aprobado & \\
\hline \multicolumn{4}{|l|}{ Matemáticas } \\
\hline Suspenso & 30 & 44 & 40.5 \\
\hline Aprobado & 14 & 188 & 93.5 \\
\hline$\%$ Global & & & 79 \\
\hline \multicolumn{4}{|l|}{ Lenguaje } \\
\hline Suspenso & 0 & 46 & 0 \\
\hline Aprobado & 2 & 229 & 99.1 \\
\hline$\%$ Global & & & 82.7 \\
\hline
\end{tabular}

Los componentes de los modelos se expresan en la Tabla 5 . En cuanto al éxito en matemáticas, la odd ratio obtenida para la variable indica que la ventaja de que los estudiantes prosociales tengan éxito en matemáticas es .36 veces menor por cada unidad que aumenta su puntuación en la subescala de atribución del fracaso en matemáticas a la baja capacidad.
Respecto al éxito en lenguaje, las odds ratio indican que: a) la ventaja de que los estudiantes prosociales tengan éxito en lenguaje es 1.61 veces mayor por cada unidad que aumenta la puntuación en la subescala atribución del éxito en lenguaje al esfuerzo y, b) la ventaja de que los estudiantes prosociales tengan éxito en lenguaje es .62 veces menor por cada unidad que aumenta la puntuación en la subescala atribución del fracaso en matemáticas a la baja capacidad.

Para la predicción de las autoatribuciones académicas de los estudiantes prosociales el éxito escolar, las categorías de la variable dependiente fueron dicotomizadas en tener todas las asignaturas aprobadas $(n=155)$ y uno o más suspensos ( $n=$ 124). Los datos han permitido crear un modelo de regresión logística que logra hacer estimaciones correctas respecto a la probabilidad de éxito académico (todas las asignaturas aprobadas) en estudiantes prosociales de E.S.O. a partir de las puntuaciones en atribuciones académicas. La Tabla 6 muestra los pasos seguidos por el modelo en la introducción de variables explicativas que han resultado significativas para la predicción de la probabilidad de éxito académico. En este sentido, el estadístico $\mathrm{R}^{2}$ de Nagelkerke estima un valor de ajuste de .153 para el modelo de éxito académico.

El modelo creado para la variable dependiente éxito académico permite una estimación correcta del 69.5\% de los casos, $\chi^{2}=33.86 ; p<.001$, entrando a formar parte de la ecuación la variable predictora atribución del éxito en matemáticas a causas externas y atribución de fracaso en matemáticas a la baja capacidad (ver Tabla 7).

Tabla 5. Resultados derivados de la regresión logística binaria para la probabilidad de éxito en matemáticas y en lenguaje.

\begin{tabular}{|c|c|c|c|c|c|c|}
\hline & $\mathrm{B}$ & E.T. & Wald & $p$ & OR & IC 95\% \\
\hline \multicolumn{7}{|l|}{ Matemáticas } \\
\hline Fracaso matemáticas capacidad & -1.04 & .15 & 45.03 & .00 & .36 & $.26-.47$ \\
\hline Constante & 4.109 & .51 & 63.30 & .00 & 60.89 & \\
\hline \multicolumn{7}{|l|}{ Lenguaje } \\
\hline Exito lectura esfuerzo & .477 & .18 & 6.60 & .010 & 1.61 & $1.12-2.31$ \\
\hline Fracaso matemáticas capacidad & -.464 & .15 & 9.32 & .002 & .62 & $.46-.84$ \\
\hline Constante & 1.206 & .85 & 1.99 & .158 & 3.33 & \\
\hline
\end{tabular}

Nota. B = coeficiente de regresión. E.T. = error estándar. $p=$ probabilidad. OR = odd ratio. I.C. $=$ intervalo de confianza al $95 \%$. 
Tabla 6. Pruebas omnibus y ajuste sobre los coeficientes del modelo rendimiento académico.

\begin{tabular}{clcccc}
\hline & Variables & $\chi^{2}$ & $G l$ & $p$ & R de Nagelkerke \\
\hline Paso 1 & Exito matemáticas externas & 26.51 & 1 & .000 & .000 \\
& Modelo & 26.51 & 1 & .121 \\
\hline Paso 2 & Fracaso matemáticas capacidad & 7.34 & 1 & .000 & .000 \\
& Modelo & 33.86 & 2 & .153 \\
\hline
\end{tabular}

Nota. $g l=$ grados de libertad. $p=$ probabilidad.

Tabla 7. Número y porcentaje de casos clasificados correctamente en la predicción del éxito académico.

\begin{tabular}{|c|c|c|c|}
\hline \multirow{2}{*}{ Observado } & \multicolumn{2}{|c|}{ Pronosticado } & \multirow{2}{*}{$\begin{array}{l}\% \text { Casos } \\
\text { acertados }\end{array}$} \\
\hline & Suspenso & Aprobado & \\
\hline Suspenso & 69 & 55 & 55.6 \\
\hline Aprobado & 30 & 125 & 80.6 \\
\hline$\%$ Global & & & 69.5 \\
\hline
\end{tabular}

Los componentes del modelo se expresan en la Tabla 8. Las odds ratio obtenidas indican que: (a) la ventaja de que los estudiantes prosociales tengan éxito académico (aprobar todas las asignaturas) es 1.64 veces mayor por cada unidad que incrementa su puntuación en la subescala de éxito en matemáticas a causas externas y, (b) la ventaja de que los estudiantes prosociales aprueben todas las asignaturas es .59 veces menor por cada unidad que aumenta su puntuación en la subescala de fracaso en matemáticas a la baja capacidad

Tabla 8. Resultados derivados de la regresión logística binaria para la probabilidad de éxito académico.

\begin{tabular}{lccccc} 
Tabla 8. Resultados derivados de la regresión logística binaria para la probabilidad de éxito académico. \\
\hline
\end{tabular}

Nota. B = coeficiente de regresión. E.T. = error estándar. $p=$ probabilidad. OR = odd ratio. I.C. $=$ intervalo de confianza al $95 \%$.

\section{Discusión}

Los resultados de este estudio indican que los estudiantes prosociales atribuyen significativamente más que los estudiantes no prosociales el éxito en lenguaje a la capacidad y al esfuerzo y, en menor medida, a causas externas, el éxito en matemáticas al esfuerzo y el fracaso en matemáticas a la falta de esfuerzo. Por el contrario, los estudiantes prosociales atribuyen significativamente menos que los estudiantes no prosociales el éxito en matemáticas a causas externas y el fracaso en lenguaje a la baja capacidad.

Los estudiantes prosociales parecen responsabilizarse más que los no prosociales de sus éxitos y fracasos académicos tanto en matemáticas como en lenguaje, al igual que lo hacen en el contexto de las relaciones sociales, confirmando cierta semejanza o paralelismo entre el contexto académico y social indicado por diversos autores (por ejemplo, Weiner, 2005; Wentzel, 2004).

Ahora bien, un análisis más profundo respecto a los tipos de causas (capacidad, esfuerzo o externas) que utilizan los estudiantes prosociales para explicar sus éxitos y fracasos en las asignaturas de matemáticas y lenguaje revela que éstos presentan un patrón atribucional adaptativo en ambas asignaturas. Concretamente, tanto en situaciones de éxito como de fracaso en matemáticas, estos estudiantes realizan atribuciones al esfuerzo empleado o a la falta del mismo, respectivamente. Además, atribuyen mucho menos los fracasos en lenguaje a la falta de capacidad que sus iguales no prosociales. Ello, sin lugar a dudas, mantendrá y potenciará la motivación, el autoconcepto y el éxito académico en ambas materias en tanto que, además de sentirse responsables de sus éxitos y fracasos atribuyéndolos al esfuerzo o a la falta del mismo, sus fracasos no serán explicados por una falta de capacidad. De esta manera, evitarán estados emocionales negativos (por ejemplo, indefensión, sentimientos de incompetencia, pérdida de confianza) y comportamientos desadaptativos (tales como la evitación y/o escape ante situaciones de evaluación), protegiendo sus percepciones de valía personal (Covington, 1984, 2000) y teniendo efectos importantes en sus expectativas de éxito y fracaso futuras como en el autoconcepto, la autoestima y el desempeño escolar de los alumnos (García-Fernández et al., 2010; Inglés, MartínezGonzález y García-Fernández, 2013).

Por otra parte, los análisis de regresión logística revelaron que la ventaja de los estudiantes prosociales de tener éxito o aprobar matemáticas $(\mathrm{OR}=-.36)$, lenguaje $(\mathrm{OR}=-.66)$ o todas las asignaturas $(\mathrm{OR}=-.59)$ fue significativamente mayor por cada unidad que disminuyó su puntuación en la subescala de atribución del fracaso a la capacidad, lo que refleja, una vez más, el patrón atribucional adaptativo de los estudiantes prosociales.

En este punto cabría preguntarse si el patrón o perfil atribucional de los estudiantes prosociales varía en función del género y el curso académico en matemáticas y leguaje, ya que, según diversos autores, el tipo de asignaturas es una variable clave que puede mediar la relación entre atribuciones causales y género (Anderman y Midgley, 1997; Patrick et al., 1999).

En este sentido, los resultados de este estudio muestran que las chicas prosociales, en comparación con los chicos prosociales, atribuyen significativamente más el éxito en lenguaje a causas externas y atribuyen el fracaso en matemáticas más a la baja capacidad, mientras que los chicos prosociales informan un nivel significativamente más alto en atribucio- 
nes de éxito en matemáticas a la capacidad y a causas externas que las chicas prosociales. Además, los resultados no revelaron diferencias de género en autoatribuciones de fracaso en lenguaje.

Ante estos resultados se podría concluir que las chicas presentan un patrón atribucional más desapatativo que los chicos en tanto que ofrecen las mismas explicaciones que los chicos ante los éxitos en ambas asignaturas y, además, atribuyen su fracaso en matemáticas a la falta de capacidad en mayor medida que los chicos. Estos resultados son consistentes con los hallados en estudios previos (Burgner y Hweston, 1993; Postigo et al., 1999; Stipek, 1984; Wiegers y Frieze, 1977). Así, los chicos pueden realzar su imagen personal (Smith, Sinclair y Chapman, 2002) ya que están más preocupados que las chicas por mostrar una imagen positiva de si mismos en la clase, por lo que tienden a buscar juicios positivos de competencia (Ryan, Hicks y Midgley, 1997). Además, este interés por realzar el yo lleva a los chicos a abordar el proceso de aprendizaje de forma superficial, haciendo poco uso de estrategias de aprendizaje significativo

\section{Referencias}

Anderman, E.M. y Midgley, C. (1997). Changes in achievement goal orientations, perceived academic competente and grades across the transition to middle level schools. Contemporary Educational Psychology, 22, 269-298.

Bell, S.M., McCallum, R.S., Bryles, J., Driesler, K., McDonald, J., Park, S.H., \& Williams, A. (1994). Attributions for academic success and failure: An individual difference investigation of academic achievement and gender. Journal of Psychoeducational Assessment, 12, 4-13.

Burgner, D. y Hewstone, M. (1993). Young children's causal attributions for success and failure: "Self-enhancing boys" and "self-derogating girls". British Journal of Developmental Psychology, 11, 125-129.

Carlo, G., Fabes, R.A., Laible, D., Kupanoff, K.I. (1999). Early adolescence and prosocial/moral behavior II: The role of social and contextual influences. Journal of Early Adolescence, 19 (2), 133-147.

Carrasco, C. y Trianes, M.V. (2010). Clima social, prosociabilidad y violencia como predictoras de inadaptación escolar en primaria. European Journal of Education and Psychology, 3(2), 229-242.

Cerezo, M. T. y Casanova, P. F. (2004). Diferencias de género en la motivación académica de los estudiantes de Educación Secundaria Obligatoria. Revista Electrónica de Investigación Educativa, 2, 97-112.

Clark, R., Dogan, R.R. y Akbar, N.J. (2003). Youth and parental correlates of externalizing symptoms, adaptive functioning, and academia performance: An exploratory study in preadolescent black. Journal of Black Psychology, 29, 210-229.

Covington, M.V. (1984). The self-worth theory of achievement motivation: findings and implications. The Elementary School Journal, 85, 5-20.

Cooley, E., Beaird, J. y Ayres, R. (1994). Academic persistence and attributional style in fifth grades. Psychology in the Schools, 31, 156-163.

Craven, R. G., Marsh, H. W. \& Debus, R. L. (1991). Effects of internally focused feedback and attributional feedback on enhancement of academic self-concept. Journal of Educational Psychology, 83, 17-27.

Crick, N.R. y Ladd, G.W. (1990). Children's perceptions of the outcomes of social strategies: Do the ends justify being mean? Developmental Psychology, 26, 612-620.

Diener, C. y Dweck, C.S. (1978). An analysis of learned helplessness: Continuous changes in performance, strategy, and achievement cognitive following failure. Journal of Personality and Social Psychology, 36, 451-462.

Dodge, K.A. (1980). Social cognition and children's aggressive behaviour. Child Development, 51, 162-170.

Dodge, K.A. y Frame, C.L. (1982). Social cognitive biases and deficits in aggressive boys. Child Development, 53, 344-353.
(Cerezo y Casanova, 2004; Valle et al., 1997). Estos resultados resultados también podrían explicar, en parte, que los chicos obtengan mejores resultados académicos en matemáticas (PISA, 2003).

En cuanto a las diferencias de curso en autoatribuciones de estudiantes prosociales, los resultados revelan que las atribuciones de fracaso a la capacidad y al esfuerzo en la asignatura de lenguaje incrementan en función del curso académico. Concretamente, las atribuciones de fracaso a la baja capacidad en lenguaje aumentan moderadamente en $4^{\circ}$ de E.S.O., mientras que atribuciones de fracaso al bajo esfuerzo incrementan moderadamente en $3^{\circ}$ de E.S.O. y este incremento se mantiene en $4^{\circ}$ de E.S.O. Además, tal y como se esperaba, las atribuciones de fracaso a causas externas en la asignatura de matemáticas disminuyen en $3^{\circ}$ de E.S.O., manteniéndose esta disminución en el siguiente curso. Finalmente, podría concluirse que conforme avanzan los cursos académicos $\mathrm{y}$, por tanto, incrementa la edad, los estudiantes realizan atribuciones más internas, responsabilizándose más ante sus éxitos y fracasos.

Dodge, K.A. (2006). Translational science in action: Hostile attributional style and the development of aggressive behavior problems. Development and Psychopathology, 18, 791-814.

Dweck, C.S. (1975). The role of expectations and attributions in the alleviation of learned helplessness. Journal of Personality and Social Psychology, 31, 674-685.

Dweck, C.S. y Leggett, E.L. (1988). A social-cognitive approach to motivation and personality. Psychological Review, 95, 256-272.

Dweck, C.S. (1996). Social motivation: Goals and social-cognitive processes. A comment. En J. Junoven y K.R. Wentzel (Eds.), Social motivation: Un derstanding children's school adjustment (pp. 181-195). New York: Cambridge University Press

Elliot, S.N. y Dweck, C.S. (1988). Goals: An approach to motivation and achievement. Journal of Personality and Social Psychology, 25, 109-116.

Entwisle, D.R., Alexander, K.L., Pallas, A.M. y Cadigan, D. (1987). The emergent academic self-image of first graders: Its response to social structure. Child Development, 58, 1190-1206.

Feshbach, N. D. y Feshbach, S. (1987). Affective processes and academic achievement. Child Development, 58, 1335-1347.

Ford, M.E. (1996). Motivational opportunities and obstacles associated with social responsibility and caring behaviour in school contexts. En J. Junoven y K.R. Wentzel (Eds.), Social motivation: Understanding children's school adjustment (pp. 126-153). New York: Cambridge University Press

García-Fernández, J. M., Inglés, C. J., Torregrosa, M. S., Ruiz-Esteban, C. Díaz-Herrero, A., Pérez-Fernández, E. y Martínez-Monteagudo, M. C. (2010). Propiedades psicométricas de la Escala de Autoeficacia Percibida Específica de Situaciones Académicas en una muestra de estudiantes españoles de Educación Secundaria Obligatoria. European Journal of Education and Psychology, 3, 61-74.

Goetz, T.S. y Dweck, C.S. (1980). Learned helplessness in social situations. Journal of Personality and Social Psychology, 39, 246-255.

Green, K.D., Forehand, R., Beck, S.J. y Vosk, B. (1980). An assessment of the relationship among measures of children's social competence and children's academic performance. Child Development, 51, 1149-1156

Gutiérrez, M; Escartí, A. y Pascual, C. (2011). Relaciones entre empatía, conducta prosocial, agresividad, autoeficacia y responsabilidad personal y social de los escolares. Psicothema, Vol. 23, no 1, pp. 13-19

Hull, J.G., Van Treuren, R.R. y Propsom, P.M. (1998). Attributional style and the components of hardiness. Personality and Social Psychology Bulletin, $14,505-513$. 
Inderbitzen, H.M. y Foster, S.L. (1992). The Teenage Inventory of Social Skills: Development, reliability, and validity. Psychological Assessment, 4, 451-459.

Inglés, C.J., Hidalgo, M.D., Méndez, F.X. e Inderbitzen, H.M. (2003). The Teenage Inventory of Social Skills: Reliability and validity of the Spanish translation. Journal of Adolescence, 26, 505-510.

Inglés, C.J., Méndez, F.X., Hidalgo, M.D., Rosa, A.I. y Estévez, C. (2003). Evaluación de las habilidades sociales en educación secundaria: Revisión de cuestionarios, inventarios y escalas. Psicología Educativa, 9, 71-87.

Inglés, C.; Benavides, G.; Redondo, J.; García-Fernández, J.M.; RuizEsteban, C.; Estévez, C. y Huescar, E. (2009). Conducta prosocial y rendimiento académico en estudiantes españoles de Educación Secundaria Obligatoria. Anales de Psicología, 25, 1, 93-101.

Inglés, C. J., Martínez-González, A. E., Valle, A., García-Fernández, J. M. \& Ruiz- Esteban, C. (2011). Conducta prosocial y motivación académica en estudiantes españoles de Educación Secundaria Obligatoria. Universitas Psychologica, 10 (2), 451-465.

Inglés, C.J.; Martínez- González, A. y García-Fernández, J. M. (2013). Conducta prosocial y estrategias de aprendizaje en una muestra de estudiantes españoles de Educación Secundaria Obligatoria. European Journal of Education and Psychology, 6 (1), 33-53.

Junoven, J. (1996). Self-presentation tactics promoting teacher and peer approval: The function of excuses and other Cléber explanations. En J. Junoven y K.R. Wentzel (Eds.), Social motivation: Understanding children's school adjustment (pp. 43-65). New York: Cambridge University Press.

Marsh, H. W. (1984). Relationship among dimensions of self-attribution, dimensions of self-concept, and academic achievements. Journal of educational psychology, 76, 1291-1308.

Marsh, H.W., Cairns, L., Relich, J., Barnes, J. y Debus, R.L. (1984). The relationship between dimensions of self-attribution and dimensions of selfconcept. Journal of Educational Psychology, 76, 3-32.

Martens, B.K. y Witt, J.C. (2004). Competence, persistence, and success: The positive psychology of behavioral skill instruction. Psychology in the Schools, 41, 19-30.

Patrick, H., Ryan, A.M. y Pintrinch, P.R. (1999). The differential impact of extrinsic and mastery goal orientations on males' and females' selfregulated learning. Learning and Individual Differences, 11, 153-171.

PISA. Características del estudio PISA 2003. Apuntes del Instituto de Evaluación. MEC. Número 03. 2006.

Postigo, Y., Pérez, M. y Sanz, A. (1999). Un estudio acerca de las diferencias de género en la resolución de problemas científicos. Enseñanza de las Ciencias, 17, 247-258.
Raine, A., Dodge, K.A., Loeber, R. et al. (2006). The reactive-proactive aggression questionnaire: Diferential correlates of reative and proactive aggression in adolescent boys. Aggressive Behavior, 32, 159-171.

Ryan, A.M., Hicks, L. y Midgley, C. (1997). Social goals, academic goals and avoiding seeking help in the classroom. Journal of Early Adolescence, 17, 152-171.

Smith, L, Sinclair, K.E. y Chapman, E.S. (2000). Students' goals, selfefficacy, self-handicapping and negative affective responses: An Australian senior school student study. Contemporary Educational Psychology, 27, 471-485.

Stipek, D. J. (1984). Sex differences in children's attributions for success and failure on math and spelling tests. Sex Roles, 11, 34-42.

Taylor, L.D., Davis, K.P. y Malanchuk, O. (2007). Self-esteem, academia self-concept, and aggression at school. Aggressive Behavior, 33, 130-136.

Vandiver, T. (2001). Children's social competence, academic competence, and aggressiveness as related to ability to make judgments of fairness. Psychological Reports, 89, 111-121.

Watkins, D. y Gutierrez, M. (1990). Causal relationships among selfconcept, attributions, and achievement in Filipo students. The Journal of Social Psychology, 130, 625-631.

Weiner, B. (1974). Achievement motivation and attribution theory. Morristown, N.J.: General Learning Press.

Weiner, B. (1986). An attributional theory of motivation and emotion. New York:Springer-Verlag.

Weiner, B. (2000). Intrapersonal and interpersonal theories of motivation from an attributional perspective. Educational Psychology Review, 12, 1-14.

Weiner, B. (2005). Motivation from an attribution perspective and the social psychology of perceived competence. En A.J. Elliot y C.S. Dweck (Eds.), Handbook of competence and motivation (pp. 73-84). New York: Guildford Press.

Wentzel, K.R. (1989). Adolescent classroom goals, standards for performance, and academic achievement: An interactional perspective. Journal of Educational Psychology, 81, 131-142.

Wentzel, K.R. (1994). Relations of social goal pursuit to social competence, classroom behaviour, and perceived social support. Journal of Educational Psychology, 86, 173-182.

Wiegers, I.M. y Friere, I.H. (1977). Gender, female traditionally, achievement level and cognitions of success and failure. Psychology of Women Quarterly, 2, 125-137.

(Articulo recibido: 6-3-2012; revisado: 21-2-2013; aceptado: 3-5-2013) 